\title{
Knowledge Based Methods and Tools for TQM in Small Batch Flexible Manufacturing and Complex Assembly
}

\author{
Dipl.-Ing. Michael Thie, Dr.-Ing. Dragan Stokic \\ ATB Institute for Applied System Technology Bremen GmbH \\ Wiener Str. 1, 28359 Bremen, Germany, Tel.: +421 220920 \\ Fax.: +4212209 210, thie@atb-bremen.de
}

\begin{abstract}
This paper presents a new approach to identify the causes of errors and to define appropriate measures to improve processes in small batch manufacturing and complex assembly. It includes a description on the state of the art, the procedure for the identification of error causes, the procedure for the definition of measures to improve processes and the implementation of tools/components which support this procedure.
\end{abstract}

\section{Keywords}

quality, error causes, measures, small batches, assembly

\section{INTRODUCTION}

Increased competition on the global market and quality requirements such as QS 9000 , VDA 6.1 force companies not only to ensure quality capable processes, but also to minimize their quality related costs. The modern TQM approach, aimed at ensuring the objective of zero defects at minimal costs, stresses the optimisation of quality aspects in the product and the production process design in an integrated fashion, rather than planning the quality aspects of the product and the production process in an isolated manner. There are clear user needs for methods as well as IT solutions which can effectively support advanced TQM concepts based on the integration of production and quality related activities.

A reduction of errors or their prevention requires efficient methods and tools to identify the causes of (potential) errors as well as to define appropriate measures to eliminate these causes. For mass, series production a number of methods such as Statistical Process Control (SPC), Dynamic Sample Control (DSC) etc. are available and being efficiently applied in industry (Maylor, 1991). However, in small

The original version of this chapter was revised: The copyright line was incorrect. This has been corrected. The Erratum to this chapter is available at DOI: 10.1007/978-0-387-35390-6_58 
batch flexible manufacturing systems (FMS) and complex assembly these methods often can not be directly applied due to the limited amount of quality data and the high complexity of assembly processes. Therefore, there is a need to develop/ enhance methods and tools for this process in small batch manufacturing and complex assembly.

This paper presents a new approach to solve the complex problems of identifying error causes and defining appropriate measures to improve the processes in small batch manufacturing and complex assembly. The approach is based on an effective combination of knowledge based and statistical methods, together with fuzzy logic and self-learning systems. The proposed approach is supported by IT-based tools which will be applied in the quality control of an FMS and complex assembly.

The results presented in the paper are part of the ongoing ESPRIT/AIT project QUETA (Quality engineering tools for assembly and small batch manufacturing) including several large companies, primarily from the automotive and aerospace industry (Daimler-Benz, Volkswagen, British Aerospace, Saab, Bosch), as well as several research partners from different EU countries (QUETA, 1997).

\section{STATE OF THE ART}

The possible approaches for the identification of error causes in small batch manufacturing and assembly are (QUETA, 1997, Al-Salti, 1994):

- Statistical Approaches (Statistical Process Control, Control Charts etc.),

- System and Model Based Approaches (Simulation, Process Parameter Comparison etc.),

- System and Knowledge Based Approaches (Rule based Systems, Fuzzy Control, Fixed assignment error-error cause, FMEA, Ishikawa method, Error tree approach etc.),

- Learning Approaches (Neural networks, Single test for defined error causes etc.).

An evaluation of the considered approaches with required criteria such as appropriateness for application on the shop-floor, efforts for creating the evaluation programs, efforts for data acquisition and processing, assumed reliability of the results, robustness etc. shows that each of the analysed approaches have certain advantages and disadvantages. For example, statistical approaches require no modelling efforts and are relatively easy to use, but the problems related to the statistical significance of small sample size and the interpretation aspects are critical. On the other hand, system and model based approaches have good interpretation possibilities and do not need high sample size, but they require high modelling efforts and are sensitive to changes in processes.

It can be concluded that there is no approach which fulfils all users requirements. This is the reason why the identification of error causes in small batch manufacturing and assembly is currently carried out in most companies without a specific systematic method and mainly based on the knowledge/experience of individual employees. This situation leads to a number of problems such as: the identification 
process is time and effort consuming, especially in the transition phase; availability of a very limited number of experts to carry out the process of identifying error causes; no organised transfer of experience from one transition process to another; no guarantee that the processes are stable, even when it seems that the 'stable phase' has been reached, etc.

However, an effective combination of different approaches allows to fulfil the specified requirements to identify error causes in small batch manufacturing and assembly and to enable the generation of cost effective activity plans for eliminating these causes.

\section{GENERAL CONCEPT / SOLUTION}

To overcome the stated problems innovative methods to identify the reasons of errors, as well as to generate action plans in order to eliminate these reasons in small batch manufacturing and assembly have been developed. The basic concept is presented in Figure 1.

- Feedback path: Identification of error causes and update of error knowledge (QUPROC), as well as generation of action plans to eliminate the identified error causes (QUPLAN).

- Feedforward path: Identification of possible error causes (QUPROC) and advance elimination (e.g. when some changes in process or product are planned etc. - QUPLAN).

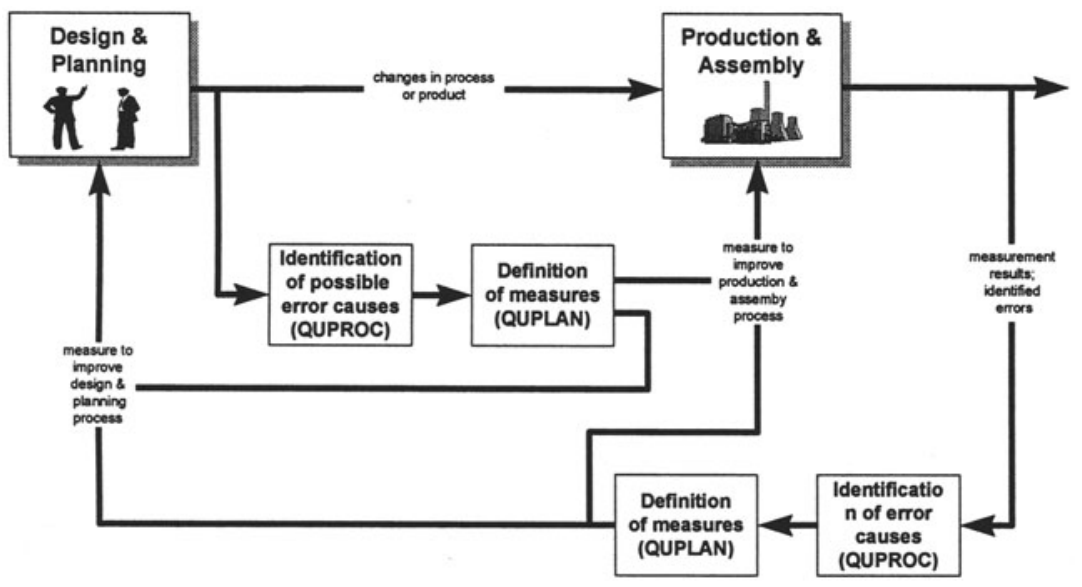

Figure 1: Basic concept for the identification of error causes and the generation of action plans.

In reference to TQM, the prevention of errors (make it right the first time) should be the basic objective in order to improve quality. However, to prevent errors, a high amount of knowledge about the specific processes and products is necessary. Practically, it is not possible to create a model in advance to identify all possible (critical) error causes because there are too many influencing factors (e.g. machine, 
environment, employees). This means that a further feedback path is required to create and update knowledge w.r.t. errors and their causes, based on the results of quality testing in the current production process. Therefore, the developed methods (and tools QUPROC and QUPLAN to support these methods) are active on both paths.

The developed methods are based on the following approaches. The main problem related to small batch manufacturing is that sample size may not be sufficient to fulfil the requirements regarding statistical significance. A possible solution is to reach acceptable sample size by producing statistics across the common features of the same or different products manufactured with the same process. To achieve these different possibilities for the correlation of features, it is necessary to consider for e.g. deviation from 'nominal dimensions' (normalised), rather than absolute values, in order to enable the calculation of statistics across common features, socalled features based batch overlapping control charts may be applied etc. (see Figure 2). The basic approach for the identification of critical processes in small batch manufacturing is to apply a combination of statistical methods, knowledge based systems to interpret statistical results, to enable the 'building' of statistically relevant samples, modelling approach restricted to simple, relatively rough modelling, learning approach restricted to updates of 'rules'. The main problems in the application of such an approach are: identifying features which can be correlated, applying a consistent and reliable set of rules for interpreting statistical results etc.

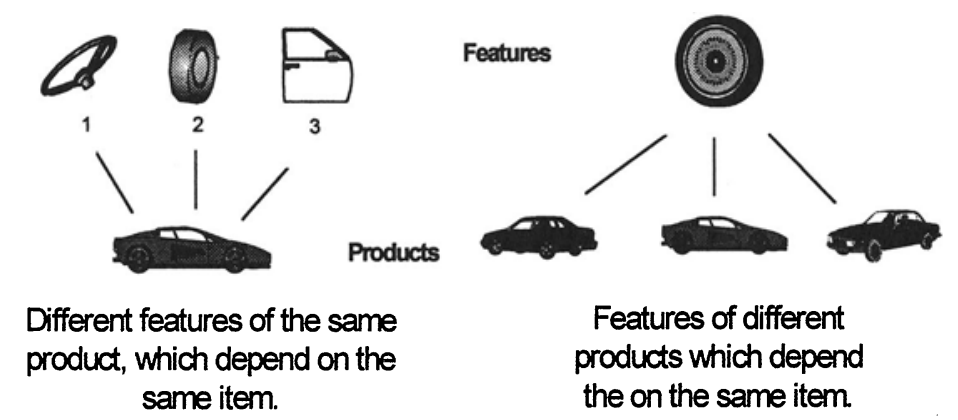

Figure 2: Basic rational for the identification of critical processes and of reasons for errors in small batch manufacturing.

Similarly, the basic approach for the identification of quality critical processes in small batch assembly is to apply a combination of knowledge based systems to capture the experts experience and know-how (transferred into a set of rules), which is supported by a statistical approach, modelling approach restricted to simple, relatively rough modelling, learning approach restricted to updates of rules and parameters (and structure) of a model. The main problems related to such an approach are: 'extrapolation' of rules to errors which have not appeared before, and the reliable and efficient capture of knowledge etc. 


\section{PROCEDURE FOR THE IDENTIFICATION OF ERROR CAUSES}

As above explained, the procedure for the identification of error causes is mainly based on a rule based system in combination with grouping techniques, fuzzy logic and learning approaches. The procedure distinguishes between (see Figure 3):

- Measurement results are available, which is normally the case in production.

- Error description is given, which is normally the case in assembly, where no or very little measurements are done.

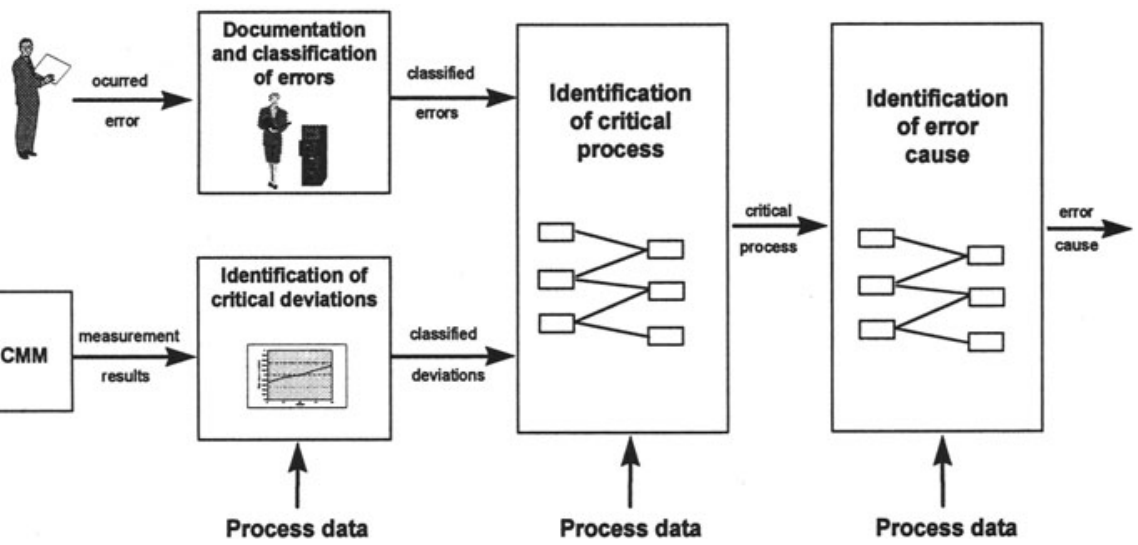

Figure 3: Procedure for the identification of error causes.

The measurement results are analysed to identify critical deviations in the process or the part. To accomplish this the measured features of the part are grouped to get a 'bigger' batch. The values of each feature are compared with the tolerances (to identify deviations in the part) and with the standard deviation (to identify deviations in the process). If a critical deviation is identified, 'a priori' rules are used to assign it to a predefined deviation/error class. The deviations are grouped/classified in such a way that each deviation in a group has similar classes of critical processes and error causes. Since it is obviously not possible to define rules valid for all possible cases additional information on the process or parts mainly in the form of statistical values are used to increase or decrease the probability of the deviation/error class. In the case that an error is identified by a user and the error description is available, the errors are classified into the predefined deviation/error classes.

If the deviation/error class for an occurred deviation/error is known the critical process/error cause has to be identified. Using defined 'a priori' rules between the deviation/error class and critical processes/error causes being applied in the manner that 'process may cause an error in an error class with a specific probability' allows for the identification of possible critical processes/error causes. The 'a priori' rules are similar as in the identification of deviation classes (see Figure 4). 


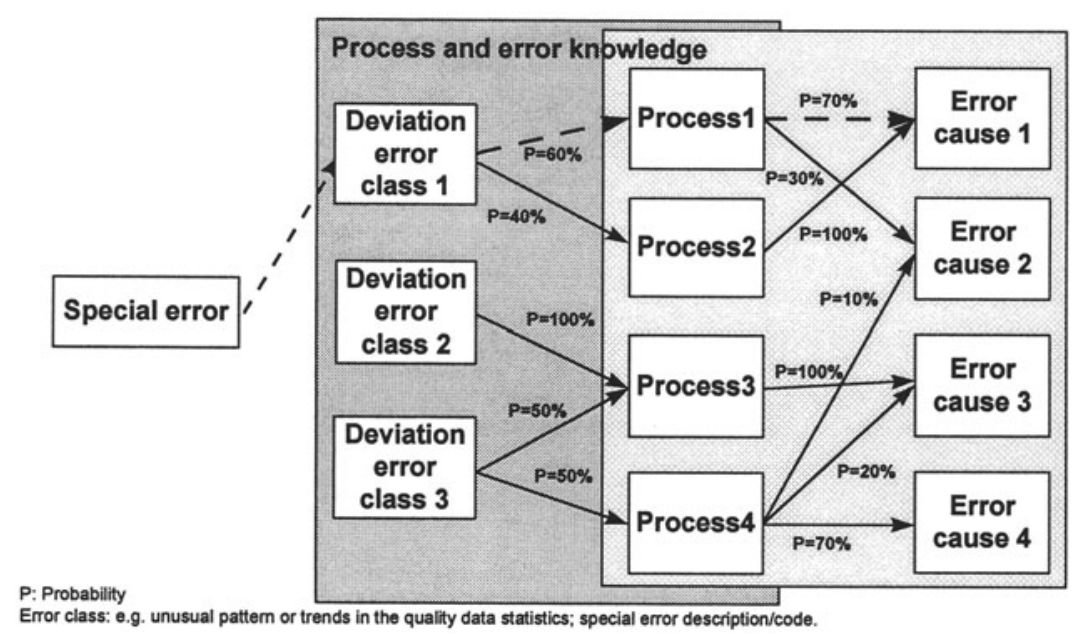

Figure 4: Relation between error/critical processes/error causes.

Since it is obviously not possible to define rules valid for all possible cases, and since using only the error description can not be sufficient to identify error causes, additional information on the procèss or products are used to increase or decrease the probability of the critical processes (see Figure 5).

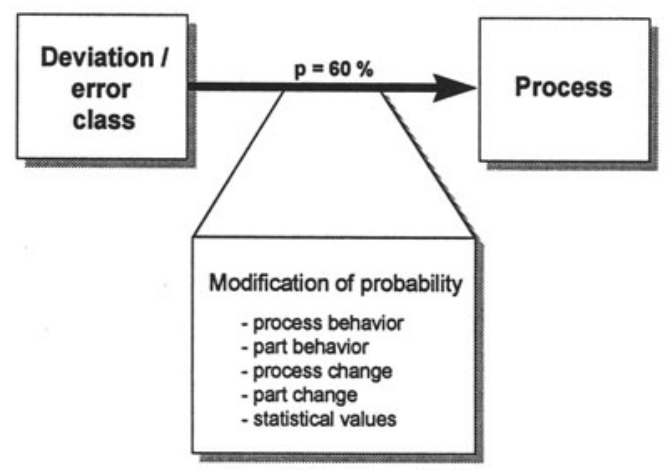

Figure 5: Modification of probabilities.

Additional information are those covering the behaviour of the process or part and changes in the process or part. In small batch manufacturing, additional information also include the results of statistical analysis. The additional information needed for a concrete application is strongly application dependent. This requires an open system which can integrate and use different kinds of information.

For integration of the additional information into the 'a priori' rules there is, additionally, a strong need to support vague terms that may appear in the premises (i.e. 'the assembly area has recently been redesigned', 'the part was for a long time in storage' etc.). An efficient way to handle such sentences is to model them via fuzzy 
sets. Fuzzy sets enable the quantitative modelling of simple sentences (e.g. 'design change is recent') consisting of linguistic variables (e.g. 'design change'), primary terms (e.g. 'recent'), and optional linguistic hedges (e.g. 'very', 'not'). To provide a basis for the model, each variable must be assigned a physical quantity (e.g. time from the moment when the design is changed) and its range of change.

The proposed method for the identification of error causes is supported by the tool QUPROC. The basic modules of this tool are presented in Figure 6. Note that a basic module of the system represents a knowledge based system which includes a set of 'a priori' rules, as well as a set of rules for handling additional information (for changing the probability of different errors).

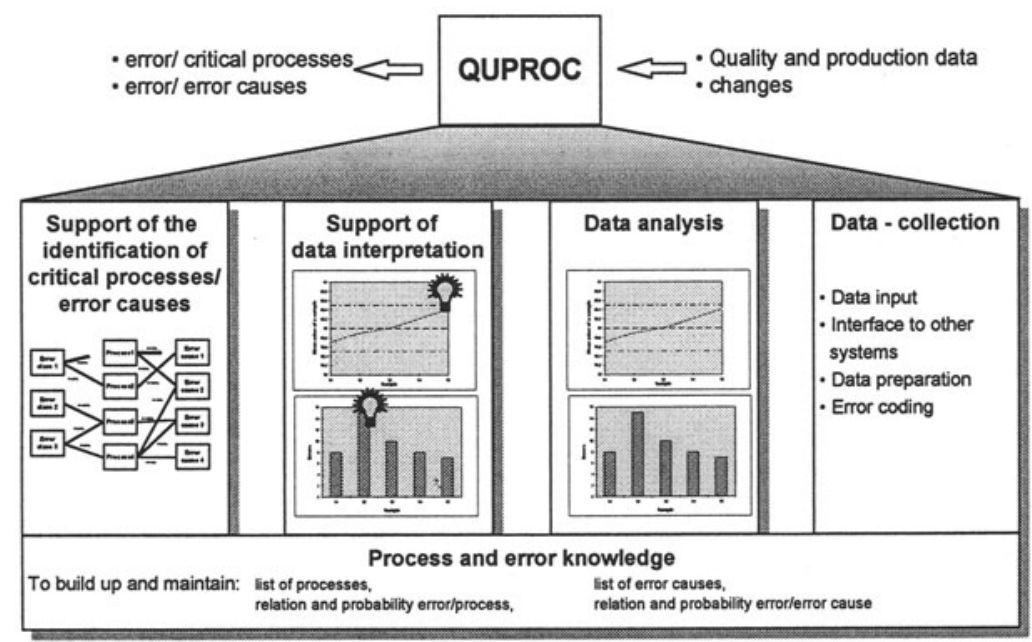

Figure 6: Modules of the tool QUPROC.

\section{PROCEDURE FOR THE DEFINITION OF MEASURES}

The results of the above explained procedure are either (a) a set of potential error causes or (b) definition of the identified error cause(s).

In the case (a) there is a need to get additional information in order to decide which of the potential error causes is actually 'responsible' for the error occurred. This may ask for additional measurements to identify the error cause (i.e. to increase the observability of the system).

In the case (b), when the actual error cause is identified, actions have to be defined as to how this error cause can be removed in order to prevent its repetition.

In both cases the procedure 'definition of measures' has to be used. These two kinds of measures (additional measurements, actions to improve the process) have to be defined based on knowledge w.r.t. possible measures in each specific case. The definition of measures is similar to the identification of error causes. The relation between error causes and measures is defined in 'a priori' rules, i.e. these rules define which of the possible actions may be appropriate for removing a specific 
error cause (or which measurements could be applied to increase the observability of the system).

However, in order to cover each specific situation, additional information on processes and products has to be used, i.e. the rules for the handling of additional information have to be applied in order to change the 'relation' between an error cause and possible measures. Applying these rules and the available additional information, it is possible to define the 'optimal' measure for an error cause ('optimal' from the viewpoint of costs, efforts and the probability that the measure will be successful).

Similarly as in the method for the identification of error causes, the method for definition of measures is supported with an appropriate tool called QUPLAN which also includes a knowledge base consisting of a set of 'a priori' rules and also rules for handling additional information.

\section{IMPLEMENTATION OF THE TOOLS/COMPONENTS}

As mentioned above in order to support these methods, the software tools QUPROC and QUPLAN are under development. As previously shown, the tools require a large amount of information which is normally available in other legacy systems of the company. To achieve this, it is necessary that the tools can be easily integrated into different hardware and software environments. They also have to run under different operating systems and have to have connections to different legacy systems.

The tools are specified and implemented with OO-Technology and integration is done using CORBA while taking into account the AIT reference architecture (AIT, 1996). Initial testing of the prototypes show promise to fulfil user requirements and considerably contribute to reducing efforts for the identification of reasons for errors, as well as increasing the efficient planning of activities to ensure quality in the scope of TQM approaches. Although the tools are primarily oriented towards the needs of the automotive and aerospace industry, it is likely that they will also be applicable in other industrial sectors.

The tools are operating in the feedback loop in order to define the measures required to improve the process based on information about occurred errors (or, deviations from nominal values), and in the feedforward path in order to define preventive measures needed to be carried out before changes in a product or in processes are executed. Within the tool set-up phase, general rules are adapted to the specific company. Based on the learning functionality of the system, the probabilities of the rules are changed based on identified error causes for specific errors. The general procedure is practically the same for small batch manufacturing and small batch assembly. The main differences are that in assembly the system uses only error information, while in manufacturing, the system uses measurement data, extracts the errors and uses statistical methods such as control charts for the identification of error causes. 


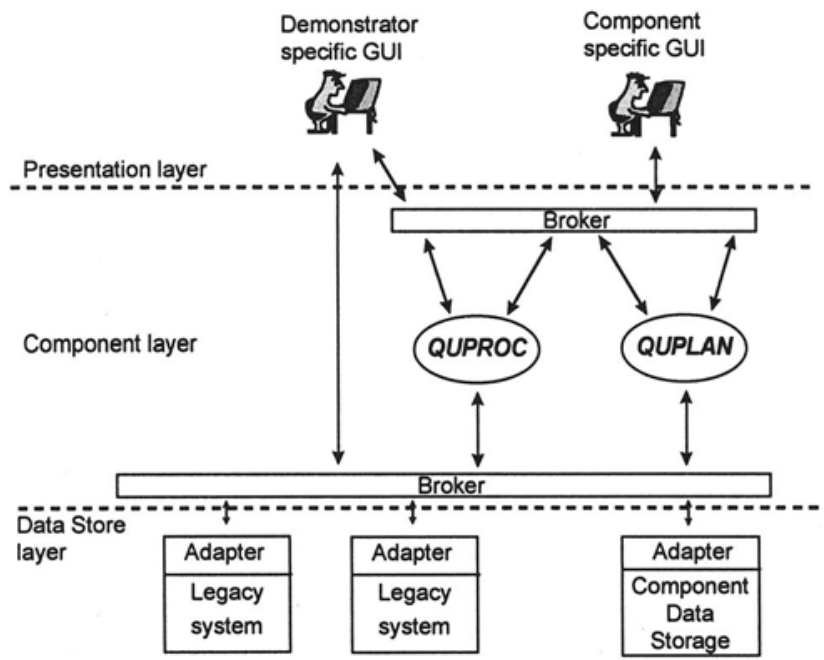

Figure 7: General architecture for the implementation of the components.

Note that the tools are supposed to work in two modes: automatic and manual. In the first case the tools enable the close of an automatic feedback quality loop, i.e. to achieve automatic control of the system w.r.t. quality. This mode is especially needed in FMS, while in the case of complex assembly the manual mode will be more appropriate, i.e. the operator has to be involved in final decisions on the error cases as well as on the measures to be applied in order to remove the reason of error (or increase the observability of the system).

\section{CONCLUSION}

The tools will be tested within different industrial demonstrator environments: i.e. in an assembly line at SAAB and in an FMS of Daimler-Benz (QUETA, 1997).

The selected demonstration area at SAAB is the assembly of tubing into the fuselage of the aircrafts carried out in the final assembly hall. The tubes are manufactured in the tubing workshop. After bending and cutting, the tubing is put into storage until needed for final assembly. At final assembly the tubing is brought from storage and installed into the fuselage. Clamping points have been provided for in sub-assembly of the fuselage that is carried out in a separate workshop. The tubing is interconnected during the installation by swaging operations and connected to the equipment by screw couplings. The installation of tubing into the fuselage caused considerable problems in the early production of certain aircraft and the problem still shows up intermittently in the stable phase of production. By applying the QUPROC and QUPLAN tools to this problem, SAAB will be able to evaluate the effectiveness of the tools by comparing results with existing experience.

In the area of replacement parts, production means or peak production capacity, as well as the initial testing w.r.t. the manufacturing of parts and the related optimisation of part design, Daimler-Benz carries out this production in job shops. A variety 
of parts are produced in such job shops with a lot size between 20 and 500. The job shop which will be used for testing of QUPROC and QUPLAN is the FMS of Daimler-Benz in Zuffenhausen. The production programme at this FMS does not allow for the use of classical methods of Statistic Process Control (SPC). Therefore, specific quality control mechanisms for small batch manufacturing are required. Up to now there are no statistical methods available to detect trends etc. Such features are urgently needed to significantly increase the economic efficiency of flexible manufacturing equipment. QUPROC and QUPLAN tools will be used to automatically close the quality control loop and enable full automatic operation of the FMS (which is specifically needed for the third - night shift).

Initial testing of the tools prototypes are very promising and it is likely that the proposed knowledge based methods will be effectively applied in these industrial systems. The selected development methodology (OO) and architecture guarantee effective and reliable application of the tools in the shop-floor conditions.

\section{REFERENCES}

Maylor, H. and Butler, C. (1991) Total Quality Control in Flexible Manufacturing Systems, in Advances in Manufacturing Technique, 491-495.

QUETA (1997) System Analysis: Analysis of users needs with respect to TQM.

Public Report, No.: PU1-03.97/1 of QUETA project, No. 22367, ESPRIT Domain 8 Task 8.5.

Al-Salti, M. and Statham, A. (1994) A review of the Literature on the use of SPC in

Batch Production. Quality and Reliability Eng. Intern., 10, 49-61. AIT (1996) IT Reference model. Deliverable of AIT-IP, No. 22148, ESPRIT

\section{BIOGRAPHY}

Dipl.-Ing. Michael Thie studied Mechanical Engineering at the University of Hannover. Since 1993 he has been working at ATB Institute for Applied System Technology Bremen GmbH. His key areas of activity are quality management, quality methods and techniques, process optimisation and introduction of ITSystems.

Dr.-Ing. Dragan Stokic worked from 1973 up to 1991 at the Institute Mihajlio Pupin in Beograd in the area of robotics and flexible automation. He was senior researcher and deputy director of the Robotics and Flexible Automation Laboratory and project manager of several projects on modelling, simulation and control of robots, flexible manufacturing systems and aircrafts. Since 1991 he has been a senior researcher at ATB Institute for Applied System Technology Bremen GmbH, responsible for research on robotics and automation, as well as on enterprise modelling and quality methods and techniques. 